\title{
Magnetically tuned spin dynamics resonance
}

\author{
J. Kronjäger ${ }^{1}$ C. Becker,${ }^{1}$ P. Navez, ${ }^{2,3}$ K. Bongs,${ }^{1}$ and K. Sengstock ${ }^{1}$ \\ ${ }^{1}$ Institut für Laserphysik, Universität Hamburg, Luruper Chaussee 149, D-22761 Hamburg, Germany. \\ ${ }^{2}$ Labo Vaste-Stoffysica en Magnetisme, Katholieke Universiteit Leuven, Celestijnenlaan 200D, B-3001 Leuven, Belgium. \\ ${ }^{3}$ additional address: Universität Duisburg-Essen, Universitätsstrasse 5, 45117 Essen, Germany
}

(Dated: September 4, 2018)

\begin{abstract}
We present the experimental observation of a magnetically tuned resonance phenomenon resulting from the spin mixing dynamics of ultracold atomic gases. In particular we study the magnetic field dependence of spin conversion in $\mathrm{F}=2{ }^{87} \mathrm{Rb}$ spinor condensates in the crossover from interaction dominated to quadratic Zeeman dominated dynamics. We discuss the observed phenomenon in the framework of spin dynamics as well as matter wave four wave mixing. Furthermore we show that the validity range of the single mode approximation for spin dynamics is significantly extended in the regime of high magnetic field.
\end{abstract}

PACS numbers: 03.75.Mn,03.75.Gg,32.60.+i

Ultracold quantum gas spin mixtures are currently receiving rapidly growing experimental and theoretical interest. They combine the unprecedented control achieved in single component Bose-Einstein condensates with intrinsic degrees of freedom. The spin-dependent coupling connects quantum gas physics to fundamental magnetic phenomena. Earlier work analyzed the basic magnetic properties of these spinor condensates [1, 2, 3, 4] and demonstrated antiferromagnetic behavior in the $F=1$ state of ${ }^{23} \mathrm{Na}[5]$ and the $F=2$ state of ${ }^{87} \mathrm{Rb}[6$ as well as ferromagnetic behavior in the $F=1$ state of ${ }^{87} \mathrm{Rb}[6$, , 7].

A particularly interesting feature of these clean and undisturbed systems is that they give access to quantum aspects of magnetism such as interaction-driven spin oscillations 6, 7, 8, 9, 10, 11, 12], the quantum classical transition [13] or domain formation [5, 12, 14]. The magnetic field dependence of the underlying spin conversion process has been analyzed in detail for $F=1$ spinor condensates [13, 15, 16, 17] and besides oscillatory behavior a resonance phenomenon was predicted [17]. Experiments so far focused on magnetic fields above the resonance 13 and on magnetic phase controlling of the spinor dynamics 12 but a spin mixing resonance has not yet been observed.

In this letter we study for the first time magnetic field tuned spin mixing in $F=2{ }^{87} \mathrm{Rb}$ spinor condensates. In particular as a main result of this letter we demonstrate resonant spin mixing behavior in spinor condensates. This resonance phenomenon becomes evident in the temporal population evolution shown in Fig. [1 As a surprising central effect, the mean field driven spin dynamics (population transfer from $m_{F} \pm 1$ to $m_{F}=0$ ) strongly depends on the external magnetic field. In contrast to studies on the natural ground state phase of the system and early findings on spin dynamics suppression [8, 9] at high magnetic field values we now find, that an external magnetic field not only leads to adverse effects but can even stimulate spin dynamics. The spin mixing amplitude is not large for zero magnetic offset field, but shows a pronounced maximum for finite magnetic field values as predicted in 13, 17]. The observation
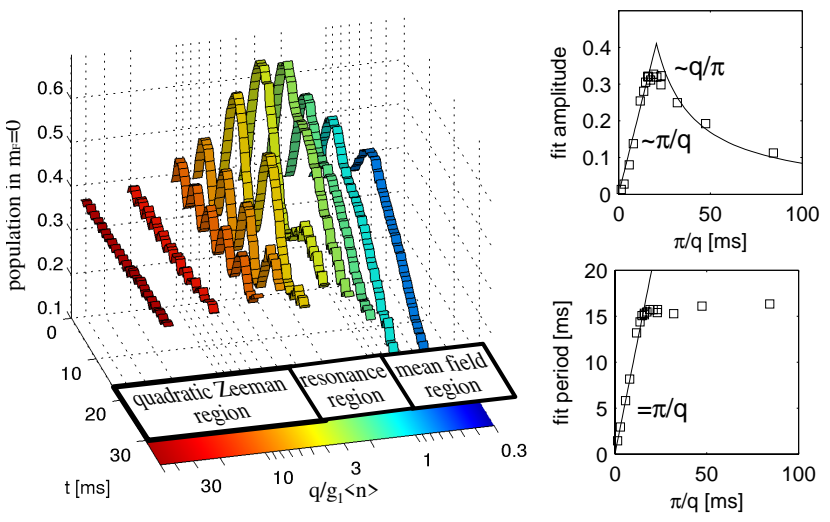

FIG. 1: Left: Temporal evolution of the $m_{F}=0$-population for different ratios of quadratic Zeeman and interaction energy (see text). Right: Magnetic field dependence of the amplitude and period of the $m_{F}=0$ population as extracted from a sinusoidal fit to the oscillatory part of the data.

and understanding of this resonance in self driven spin dynamics in quantum gases is central for detailed magnetic state manipulation. A quantum control for general spin systems (for $\mathrm{F}=1$ see [12]) based on this spinor resonance process might lead to new schemes for spin entanglement and quantum information applications with spinor gases.

The experimental setup has been described in $[\underline{6}]$ and the procedure resembles the one in 13. We prepare degenerate ${ }^{87} \mathrm{Rb}$ ensembles in the $F=2, m_{F}=-2$ state containing typically $3 \cdot 10^{5}$ atoms in an optical dipole trap (trapping frequencies $\omega_{x}: \omega_{y}: \omega_{z} \approx 2 \pi \cdot 14,5$ : $\left.105: 600 s^{-1}\right)$. A radio frequency $\pi / 2$-pulse of typically $40 \mu$ s duration rotates this state to our initial state $\zeta^{\text {ini }}(0)=(1 / 4,1 / 2, \sqrt{6} / 4,1 / 2,1 / 4)^{T}[26$. After holding the sample for a variable time, $t$, at a well defined magnetic offset field we switch off the trapping potential. The population in each spin component is extracted from absorption images after Stern-Gerlach separation during 
time of flight [5, 6]. Fig. [1] shows the temporal evolution of the $m_{F}=0$-population in dependence of the relative value of the quadratic Zeeman frequency, $q$ and the mean field spin mixing frequency, $g_{1}\langle n\rangle$. The magnetic field dependence is parameterized to first order by $q=p^{2} / \omega_{\mathrm{hfs}}$ with $p=\mu_{B} B / 2 \hbar$ and the ${ }^{87} \mathrm{Rb}$ ground state hyperfine splitting $\omega_{\mathrm{hfs}} \approx 2 \pi \cdot 6.835 \mathrm{GHz}$. The spin dependent mean field interaction is proportional to the density, $n$, of the sample and for ${ }^{87} \mathrm{Rb}$ dominated by the parameter $g_{1}$ introduced below.

Our observations show that the whole spin mixing dynamics depends critically on the relative size of the energies associated to the quadratic Zeeman shift and the spin dependent mean field interaction. Interestingly the oscillation amplitude in Fig. 1 is reduced whenever one of these energies dominates, i.e. in both the quadratic Zeeman regime $\left(q>>g_{1}\langle n\rangle\right)$ and the mean field regime $\left(q<<g_{1}\langle n\rangle\right)$. A maximum occurs close to balanced quadratic Zeeman and mean field contributions. The important point is, that these two effects compete with each other. The quadratic Zeeman effect lifts the energy of the $m_{F}=0$ state with respect to the other states, while the mean field interaction lowers it. More precisely the phase evolution due to these energy contributions is occurring in opposite directions. However,as spin mixing is a coherent process, this phase determines the direction of spin mixing. The accumulation of many particles in one state can thus only happen for slow phase evolution, i.e. close to the balanced case.

We want to emphasize, that the many-body spin mixing resonance presented in this letter crucially relies on nonlinear many-particle effects like self- and crossphase modulation. It is therefore fundamentally different from the "Rabi"-resonance case observed in spinchanging two-particle collisions [1], in which these effects are absent.

In the following we will begin our analysis of the observed spin mixing resonance phenomenon with a discussion of the the limiting cases. In particular we present and interpret analytic solutions for the asymptotic behavior in these regimes. Our analysis is based on the evolution due to the mean field hamiltonian developed in [4] using the single mode approximation (SMA).

Spin mixing occurs as a consequence of two-particle collisions and can be classified by the total spin $f$ of the colliding pair. For $F=2$ the allowed values are $f=0,2,4$. The spin mixing interactions are parameterized by the coupling coefficients $g_{1}=\frac{4 \pi \hbar^{2}}{m} \frac{a_{4}-a_{2}}{7}$ and $g_{2}=\frac{4 \pi \hbar^{2}}{m} \frac{4}{5} \frac{7 a_{0}-10 a_{2}+3 a_{4}}{7}$ [4], using the spin-dependent s-wave scattering lengths $a_{f}$. The dynamics of $F=2$ spinor condensates will in general be a complex superposition of the different mixing processes summarized in table (we use the notation $(i)$ for waves/particles in the $m_{F}=i$ state). This table also lists the relative quadratic Zeeman energy shifts of the involved states. ${ }^{87} \mathrm{Rb}$ has the advantage that the value of $g_{2}$ is very small [18], such that the processes listed in the lower part of table \is largely suppressed and can be neglected in the following [27]. In
TABLE I: Four wave mixing processes in $F=2$ spinor BEC.

\begin{tabular}{lcr}
\hline \hline process & coupling & q.Z. energy diff. \\
\hline$(0)+(0) \leftrightarrow(+1)+(-1)$ & $g_{1}$ & $2 \mathrm{q}$ \\
$(+1)+(+1) \leftrightarrow(+2)+(0)$ & $g_{1}$ & $4 \mathrm{q}$ \\
$(-1)+(-1) \leftrightarrow(0)+(-2)$ & $g_{1}$ & $4 \mathrm{q}$ \\
$(+1)+(-1) \leftrightarrow(+2)+(-2)$ & $g_{1}$ & $6 \mathrm{q}$ \\
\hline$(0)+(0) \leftrightarrow(+1)+(-1)$ & $g_{2}$ & $2 \mathrm{q}$ \\
$(+1)+(-1) \leftrightarrow(+2)+(-2)$ & $g_{2}$ & $6 \mathrm{q}$ \\
$(0)+(0) \leftrightarrow(+2)+(-2)$ & $g_{2}$ & $8 \mathrm{q}$ \\
\hline \hline
\end{tabular}

addition we will concentrate on the specific initial state $\zeta^{\text {ini }}(0)$ used in our experiments. For the population evolution in the limit of low magnetic field $\left(q<<g_{1}\langle n\rangle\right)$, i.e. the "mean field region", we find to first order:

$$
\begin{aligned}
\left|\zeta_{0}^{m f}\right|^{2} & =\frac{3}{8}\left(1-\frac{q}{2 g_{1}\langle n\rangle}\left[\cos \left(4 g_{1}\langle n\rangle t\right)-1\right]\right) \\
\left|\zeta_{ \pm 1}^{m f}\right|^{2} & =\frac{1}{4}\left(1+\frac{q}{2 g_{1}\langle n\rangle}\left[\cos \left(4 g_{1}\langle n\rangle t\right)-1\right]\right) \\
\left|\zeta_{ \pm 2}^{m f}\right|^{2} & =\frac{1}{16}\left(1-\frac{q}{2 g_{1}\langle n\rangle}\left[\cos \left(4 g_{1}\langle n\rangle t\right)-1\right]\right)
\end{aligned}
$$

This mean field dominated evolution is just a sinusoidal oscillation between the spin components and shows a magnetic field independent period $T_{m f}=\frac{\pi}{2 g_{1}\langle n\rangle}$ as observed in Fig. 1 Furthermore these equations indicate that the oscillation amplitude in this regime should be proportional to the quadratic Zeeman shift $q$, which is reflected in Fig. 1]

In the other limit at high magnetic field, i.e. the quadratic Zeeman regime with $q>>g_{1}\langle n\rangle$, the population evolution is dominated by the quadratic Zeeman energy and we find:

$$
\begin{aligned}
\left|\zeta_{0}^{q Z}\right|^{2}= & \frac{3}{8}\left\{1-\frac{g_{1}\langle n\rangle}{2 q}\left[2(\cos (2 q t)-1)+\frac{\cos (4 q t)-1}{2}\right]\right\} \\
\left|\zeta_{ \pm 1}^{q Z}\right|^{2}= & \frac{1}{4}\left\{1+\frac{g_{1}\langle n\rangle}{2 q}\left[\frac{3(\cos (2 q t)-1)}{4}-\frac{\cos (6 q t)-1}{12}\right]\right\} \\
\left|\zeta_{ \pm 2}^{q Z}\right|^{2}= & \frac{1}{16}\left\{1+\frac{g_{1}\langle n\rangle}{2 q}\left[\frac{3(\cos (4 q t)-1)}{2}\right.\right. \\
& \left.\left.+3(\cos (2 q t)-1)+\frac{\cos (6 q t)-1}{3}\right]\right\}
\end{aligned}
$$

The oscillation amplitude in this regime decays with $\frac{1}{q}$ in accordance to the data in Fig. 1] However now several frequencies are involved as a result of the different mixing processes in $F=2$ spin dynamics. The oscillation periods reflect the different quadratic Zeeman shifts for the possible $g_{1}$ mixing processes as listed in table 【 For the chosen initial state, spin mixing is dominated by the $\cos (2 q t)$ term in Eq. 2 connected to the $(0)+(0) \leftrightarrow(-1)+(+1)$ process, which has a factor of four higher weight than the $\cos (4 q t)$ term.

From the above tendencies in the population oscillation, it is clear, that a maximum amplitude must occur 
at an intermediate magnetic field value, i.e. the resonance found in Fig. 1 ]

In the following we will develop some more qualitative physical insights by an interpretation of the resonance phenomenon in terms of "phase matching" in four wave mixing (see Fig. 2). First we note that given the prefactor $\frac{1}{16}$ the oscillation amplitude of the $m_{F}= \pm 2$ wave is relatively small in both the mean field regime and the quadratic Zeeman regime. This justifies an approximate view as a pure $(-1)+(+1) \leftrightarrow(0)+(0)$ four wave mixing process. In contrast to nonlinear optics or four wave mixing in single component condensates [19] we do not deal with wavevector or momentum modes [20, 21] but with spin modes, i.e. here the $m_{F}=+1$ wave and the $m_{F}=-1$ wave couple to two times the $m_{F}=0$ wave (this view is justified, as we are considering trapped samples in a single momentum state). Spin mixing in trapped samples corresponds to degenerate collinear four wave mixing [21] and (in contrast to single component four wave mixing) in principle allows infinite interaction times, only limited by finite temperature effects [9, 13, 22, 23, 24].

In view of the optical analogy it is obvious, that phase matching considerations are essential to understand the resonance in spinor four wave mixing. The important point is, that the value of the relative phase of the initial and final waves/spin components determines the direction of wave mixing. In our case the (0) wave will be populated if the combined phase of the $(+1)+(-1)$ waves is ahead of twice the $(0)$ wave phase, i.e. $\theta=$ $\varphi_{+1}+\varphi_{-1}-2 \varphi_{0} \in[0 . . \pi]$ and it will be depopulated, if $\theta \in[\pi . .2 \pi]$ (modulo $2 \pi$ ).

In general the relative phase evolution of the spinor components in spin mixing is highly nontrivial, as in addition to the quadratic Zeeman energy shifts it depends on the spin coupling and the spin component populations in a nonlinear way. We find that for our system, the competition between mean field driven dephasing (tending to decrease $\theta$ ) and quadratic Zeeman shift driven dephasing (tending to increase $\theta$ ) determines the evolution of the system. Most importantly the evolution depends on the relative size of the quadratic Zeeman energy shift and the maximally achievable mean field shifts, as shown in Fig. 2 For large magnetic fields, i.e. always negligible mean field energy shifts, $\theta$ will continuously grow, i.e. the $( \pm 1)$ wave evolves faster than the $(0)$ wave, and the population transfer shows an oscillatory behavior depending on which wave is lagging behind at which instant (Fig. 27). In this regime the oscillation period is expected from Eq. 11 to be given by $\frac{\pi}{q}$. Furthermore the oscillation amplitude, which depends on the unidirectional mixing time, should be proportional to this period. Both these expectations are confirmed by the data, as shown in the inset of Fig. 1]

For small magnetic fields the mean field energy shift grows with increasing population in the (0) wave, until it exceeds the quadratic Zeeman shift and thus reverses the evolution of $\theta$, i.e. the (0) wave will speed up and

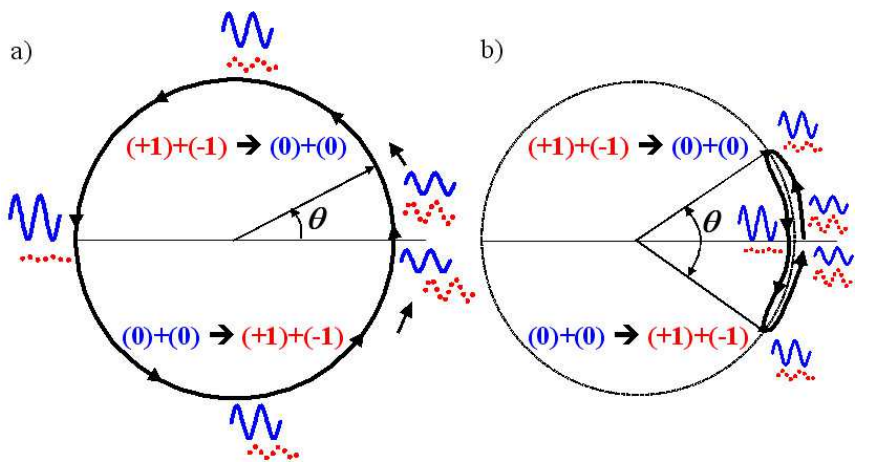

FIG. 2: Schematic representation of four wave mixing in a) the quadratic Zeeman regime and b) the mean field regime. The direction of spin mixing is indicated in the upper and lower half of the circle symbolizing the relative phase $\theta$. For $\theta>0$ the (0) waves (upper waves) get populated, while for $\theta<0$ the $(+1)$ and $(-1)$ wave (lower waves) increase.

decrease its lag behind the $( \pm 1)$ wave, eventually overtaking it. In this case $\theta$ will remain confined in the interval $[-\pi . . \pi]$ and oscillate around $\theta=0$ ( Fig. 2b). In this regime the population oscillation amplitude is expected to decrease with decreasing $q$, i.e. at lower magnetic field, as less population transfer is necessary to create a mean field energy of the size of the quadratic Zeeman shift. This decrease in amplitude is also confirmed by the data in Fig. 11.

In the resonance region at intermediate magnetic field, the relative movement of the $(0)$ and $( \pm 1)$ waves is very slow, with $\theta$ being on the border of oscillation and continuous increase. This leads to long time unidirectional spin mixing and thus maximum amplitude.

We want to point out that also the initial direction of spin mixing increasing the (0) wave amplitude as observed in Fig. 1 can be explained by simple arguments. The chosen initial state $\zeta^{\text {ini }}$ has zero phase shift $\theta=0$ and shows no mean field dephasing between the $( \pm 1)$ and (0) waves. This is due to the fact, that it originates from the fully stretched $(-2)$ eigenstate subjected to a 90 degree rotation). As the mean field part of the Hamiltonian is invariant under rotations $\zeta^{\text {ini }}$ stays a mean field eigenstate. Consequently the quadratic Zeeman energy shift will always cause the $( \pm 1)$ wave to evolve faster then the (0) wave or in other words $\theta$ will initially always grow. The transfer of particles going in the direction of the wave lagging behind will thus result in an initial increase of the (0) wave (see Fig. 2).

In addition to the understanding of the tendencies observed in Fig. 11 within the single mode approximation, a quantitative comparison allows to deduce the validity limits of this approximation for the elongated trap geometry used in the experiment. The breakdown of the single mode approximation is accompanied by the strong damping of the oscillation at low $q$, which is probably due to the onset of spatial structure formation in spin dynamics [10]. In contrast to the quadratic Zeeman dominated 

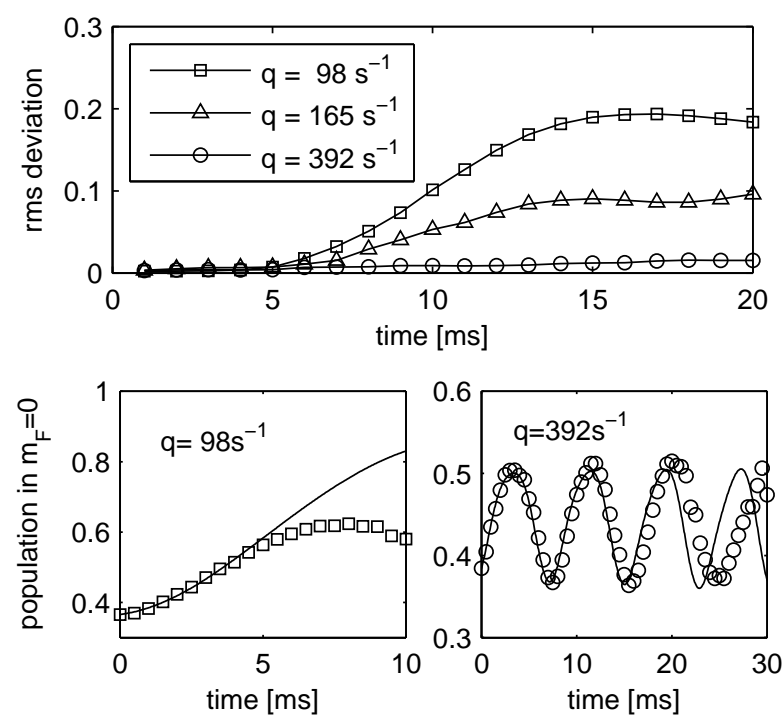

FIG. 3: TOP: Deviation (rms of $m_{F}=0$ population) of SMA theory fit $\left(g_{1}\langle n\rangle=54 \mathrm{~s}^{-1}\right.$ fixed) from our data (Fig. [1). The abscissa specifies the time range over which the fit was perfomed. BOTTOM: two examples of fits compared to data.

regime spin dynamics in the mean field regime is characterized by a density dependent coupling constant $g_{1}\langle n\rangle$. For mean field driven spin dynamics the higher density parts of the spinor condensate will thus dephase relative to the lower density parts and population oscillations in the total fractions will be washed out.

The validity range of the SMA can also be inferred from our experimental data (Fig. 3). While the evolution of Spinor condensates in our elongated geometry starts to deviate from SMA behavior after $\approx 5 \mathrm{~ms}$ in the mean field regime, there is a near perfect agreement over more than $20 \mathrm{~ms}$ in the mean field region. Our most important observation in this respect is that even for condensate sizes much above the spin healing length the single mode approximation works remarkably well once the quadratic Zeeman effect dominates the relative phase evolution.

In conclusion we have investigated a magnetically tunable resonance in coherent spin dynamics and have analyzed this phenomenon in terms of phase matching considerations, which are applicable to general values of total spin. In the future this resonance will allow the controlled use of spin dynamics to fully convert one spinor state into another, even with local control by shifting in and out of the validity range of the single mode approximation. These possibilities open new perspectives for spatiotemporal tailoring of the spinor condensate wavefunction, e.g. for the creation of highly nonclassical spin states with spatially varying degree of entanglement.

This work was funded in part by Deutsche Forschungsgemeinschaft (DFG) within SPP 1116. P.N. acknowledges support from the German $\mathrm{AvH}$ foundation and from the Junior Fellowship program of the KULeuven Research Council.
[1] T.-L. Ho, Phys. Rev. Lett. 81, 742 (1998).

[2] T. Ohmi and K. Machida, J. Phys. Soc. Jpn. 67, 1822 (1998).

[3] C. K. Law, H. Pu, and N. P. Bigelow, Phys. Rev. Lett. 81, 5257 (1998).

[4] M. Koashi and M. Ueda, Phys. Rev. Lett. 84, 1066 (2000).

[5] J. Stenger et al., Nature 396, 345 (1998).

[6] H. Schmaljohann et al., Phys. Rev. Lett. 92, 040402 (2004).

[7] M.-S. Chang et al., Phys. Rev. Lett. 92, 140403 (2004).

[8] T. Kuwamoto et al., Phys. Rev. A 69, 063604 (2004).

[9] H. Schmaljohann et al., Appl. Phys. B 79, 1001 (2004).

[10] J. M. Higbie et al., Phys. Rev. Lett. 95, 050401 (2005).

[11] A. Widera et al., Phys. Rev. Lett. 95, 190405 (2005).

[12] M.-S. Chang et al., Nature Physics 1, 111 (2005).

[13] J. Kronjäger et al., Phys. Rev. A 72, 063619 (2005).

[14] L. E. Sadler et al., cond-mat/0605351.

[15] H. Pu, S. Raghavan, and N. P. Bigelow, Phys. Rev. A 61, 023602 (2000).

[16] D. R. Romano and E. J. V. de Passos, Phys. Rev. A 70, 043614 (2004).
[17] W. Zhang et al., Phys. Rev. A 72, 013602 (2005).

[18] A. Widera et al., cond-mat/0604038.

[19] L. Deng et al., Nature 398, 218 (1999).

[20] J. P.Burke et al., Phys. Rev. A 70, 033606 (2004).

[21] E. V. Goldstein and P. Meystre, Phys. Rev. A 59, 3896 (1999).

[22] H. J. Lewandowski et al., Phys. Rev. Lett. 91, 240404 (2003).

[23] M. Erhard et al., Phys. Rev. A 70, 031602 (2004).

[24] J. Mur-Petit et al., Phys. Rev. A 73, 013629 (2006).

[25] H. Saito and M. Ueda, Phys. Rev. A 72, 053628 (2005).

[26] We use the notation $\psi=\sqrt{n}\left(\zeta_{+2}, \zeta_{+1}, \zeta_{0}, \zeta_{-1}, \zeta_{-2}(t)\right)^{\top}$ for the vector order parameter of the spinor condensate.

[27] We experimentally checked the validity of this assumption by confirming that the initial state $\zeta^{(0)}(0)=$ $(\sqrt{6} / 4,0,-1 / 2,0, \sqrt{6} / 4)^{T}$ shows no visible spin mixing within the first $15 \mathrm{~ms}$, which is the relevant timescale for coherent evolution in our experiment [13]. For this initial state all $g_{1}$-couplings vanish and the remaining coupling $(0)+(0) \leftrightarrow(+2)+(-2)$ only depends on $g_{2}[25]$. 